\title{
Low level nitrogen laser therapy in pulmonary tuberculosis
}

\author{
NS Bhagwanani, C.C. Bhatia, N. Sharma, N. Hemvani, D. S. Chitnis
}

Choithram Hospital and Research Centre, Indore (M.P)

\begin{abstract}
Background and aims: WHO estimated 9 million new Tuberculosis cases and 1.5 million TB deaths in 2013. Globally 480000 Multi drug resistant tuberculosis cases were noted and majority of them were in India, China and Russian federation. Multi drug resistant tuberculosis cases are difficult to treat and have high mortality. Presently, it was aimed to assess prevalence of drug resistance in $M$. tuberculosis isolates in Central India, to check the in-vitro effect of N2 Laser on $M$. tuberculosis and to study the therapeutic effect of intra cavitary N2 laser on pulmonary Tuberculosis cases not responding to chemotherapy.

Materials and methods: Drug sensitivity testing was carried out on 567 isolates of $M$. tuberculosis by proportion method. To check the effect of N2 laser on $M$. tuberculosis, suspension spread on LJ plate and part of the plate exposed to N2 laser for $10 \mathrm{~min}$ and plate incubated for 4 weeks to see the effect. For exposure to lung cavity a needle was introduced into the lung through which fiber was passed to the cavity for N2 laser irradiation for $10 \mathrm{~min}$.

Results: Only $12.8 \%$ isolates of $M$. tuberculosis were sensitive to all anti-Tubercular drugs and $21.5 \%$ were found to be resistant to Rifampicin qualifying definition of Multi drug resistant tuberculosis. Bactericidal effect for N2 laser was seen in-vitro on exposure to N2 laser.

Clinical improvement occurred in $90 \%$ of the 96 patients; $60 \%$ of the patients showed improvement on their X-rays and $75 \%$ turned out to be Acid fast bacilli smear negative in 4 to 15 days.

Conclusions: Intra-cavitory N2 laser therapy was found to have remarkable success as an adjunct to chemotherapy.
\end{abstract}

Key words: Tuberculosis $\cdot$ Nitrogen Laser $\cdot$ Treatment $\cdot$ Multi drug resistant tuberculosis

\section{Introduction}

Tuberculosis (TB) remains a major global health problem. It causes ill-health among millions of people each year and ranks as the second leading cause of death from an infectious disease worldwide. The latest estimate counted 9 million new TB cases in 2013 and 1.5 million TB deaths (just under 1.1 million among HIVnegative people and 0.3 million HIV-associated TB deaths). These deaths included 510000 among women and 80000 among children. There were approximately 210000 deaths from Multi drug resistant (MDR)-TB (range, 130 000-290 000). 1)

\section{Addressee for Correspondence:}

Air Commodore NS Bhagwanani,

Ex. MD Choithram, Hospital Research Centre

521, Sindh Housing Society,

Baner Road, Aundh, Pune - 411007. INDIA

Telephone: 020-25851994

E-mail: nbhagu@gmail.com
India is having the largest load of TB cases with an estimate of 2.0 million-2.3 million cases (i.e., one third of the world load). Globally an estimate of the burden of MDR-TB was 480,000 new cases of MDR-TB in 2013 and more than half of these cases were in India, China and Russian Federation. This total includes cases of primary and acquired MDR-TB. ${ }^{1)}$

The emergence of multidrug-resistant tuberculosis (MDR-TB) and, more recently, extensively drug-resistant TB (XDR-TB) is a major threat to global TB control since the therapeutic cure rate is low in such cases.

The first goal in the control of tuberculosis has to have rapid conversion to acid-fast bacillus (AFB) smear negativity of TB patient since every open case of TB is likely to generate eight more cases of tuberculosis in one year.

One of the ways could be to have alternative

Received date: March 9th, 2015

Accepted date: September 2nd, 2015 
therapeutic approach such as low level laser therapy (LLLT). Nitrogen laser has been shown to have direct anti-bacterial acticity 2,3$)$ and also accelerates intracellular killing of phagocytosed bacteria within the macrophages. ${ }^{4)}$ Macrophage activation appears to be necessary for killing of intracellular organisms. ${ }^{5)}$ The activated macrophages produce cytokines like TNFalpha and GM-CSF which inhibit growth of M. tuberculosis within macrophages. ${ }^{6-11)}$ The efficient intracellular killing of phagocytosed $M$. tuberculosis seems to be the hallmark in the control of tuberculosis.

In view of the above, it was aimed to determine the prevalence of drug resistance among $M$. tuberculosis isolates in central India, to check in-vitro effect of N2-laser on $M$. tuberculosis and to study effectiveness of intra cavitary exposure to N2 laser for pulmonary TB cases.

\section{Materials and method}

\section{Population}

Five hundred and sixty seven cases of acid-fast bacillus (AFB) smear-positive pulmonary $\mathrm{TB}$ were referred to the Microbiology Laboratory of Choithram Hospital and Research Centre (CHRC), Indore, India during the period January 1991 to August 1994. Of these cases, 62\% were on anti-TB treatment and 40\% had received irregular treatment for more than 2 years and showed an inadequate therapeutic response. The proportion of males was $65 \%$ and cases were aged in the range of 18 - 65 years.

\section{AFB culture and Sensitivity: Samples}

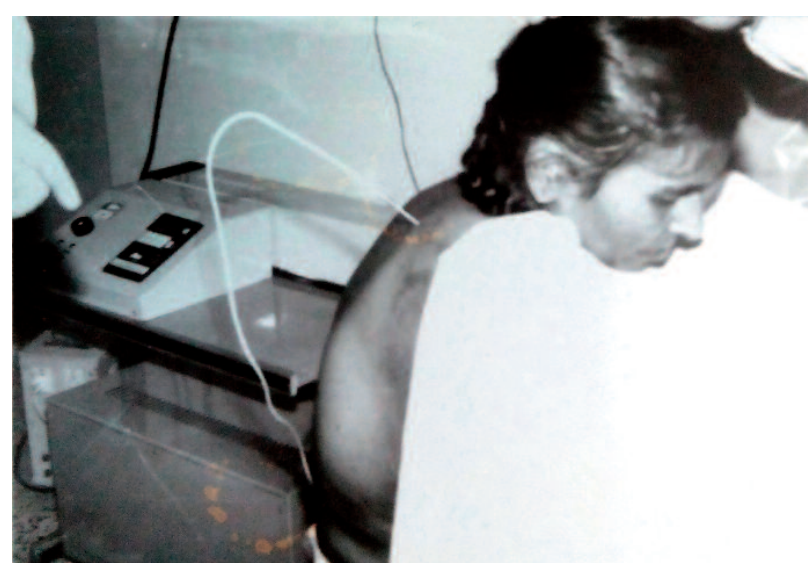

Fig. 1: Application of intra Cavitary N2 Laser on Patient.
Sputum samples were collected in wide-mouth sterile containers.

AFB smear by Auramine O fluorescent Method: Sputum smears were checked by auramine O fluorescent method for detection of AFB. ${ }^{12)}$

\section{AFB Culture:}

The sputum samples were digested, decontaminated \& concentrated by standard procedure of $\mathrm{NaOH}$ - NALC method, ${ }^{13)}$ which is also recommended by Centers for Disease control (CDC).

\section{Drug susceptibility testing by proportion method:}

The proportion method ${ }^{14)}$ was used to check the susceptibility of $M$. tuberculosis isolates using L-J medium.

In-vitro effect of N2 Laser on the growth of $M$. tuberculosis. The lawn of M. tuberculosis was made on Lowenstein Jensen Medium petri plates and the central part of plates was exposed to Nitrogen laser $5 \mathrm{~mW}$ for $10 \mathrm{~min}$. Then the plates were incubated at $37^{\circ} \mathrm{C}$ for 4 weeks.

Intra cavitary N2 laser exposure for pulmonary tuberculosis cases: The cavity was localized by x-ray. A wide bore needle, attached to a syringe filled with normal saline, was introduced into the cavity through the chest wall after local anesthesia. A free flow of air in the syringe confirmed the entry into the cavity. The syringe was disconnected and a quartz fiber from the N2 Laser (0.5 $\mathrm{mm}$ dia) was introduced through the needle into the lung cavity, which was irradiated for $10 \mathrm{~min}$ only (Fig. 1). Sometimes when the cavity is large and thick walled and there are more than one cavity, a second sitting was repeated after 3-4 days. There were no side effects from the laser. Table 3 indicates the age range and sex distribution of the patients that were treated.

Table 1: Drug resistance for ant-TB drugs (1991-1994)

\begin{tabular}{lcc}
\hline Drug resistance for & Number of Isolates & $\%$ \\
\hline One drug & 139 & 24 \\
\hline Two drug & 146 & 26 \\
\hline Three drugs & 118 & 20 \\
\hline Four or more drugs & 89 & 15.6 \\
\hline Sensitive to all & 73 & 12.8 \\
\hline TOTAL & 567 & \\
\hline
\end{tabular}


All 96 patients included in the N2 laser exposure study were sputum AFB positive, had pulmonary cavities and had taken anti-TB drugs for some time with no relief. The follow-up protocol required all patients to be on anti-TB drugs for 9 months. Sputum was checked for AFB smear and culture monthly for 9 months and had a chest X-ray monthly for 9 months. The study protocol was explained to all the patients and a written consent for participation in the study was obtained. The study was approved by the scientific committee of the hospital.

The Nitrogen laser has the following characteristics.

$\begin{array}{ll}\text { Wavelength } & 337 \mathrm{~nm} \text {, harmless UV-A range } \\ \text { Rept rate } & 100 \mathrm{~Hz} \\ \text { Pulse energy } & 20-30 \mu \mathrm{J} \\ \text { AV power } & 2 \mathrm{~mW} \\ \text { Pulse duration } & 5 \text { nanosec }\end{array}$

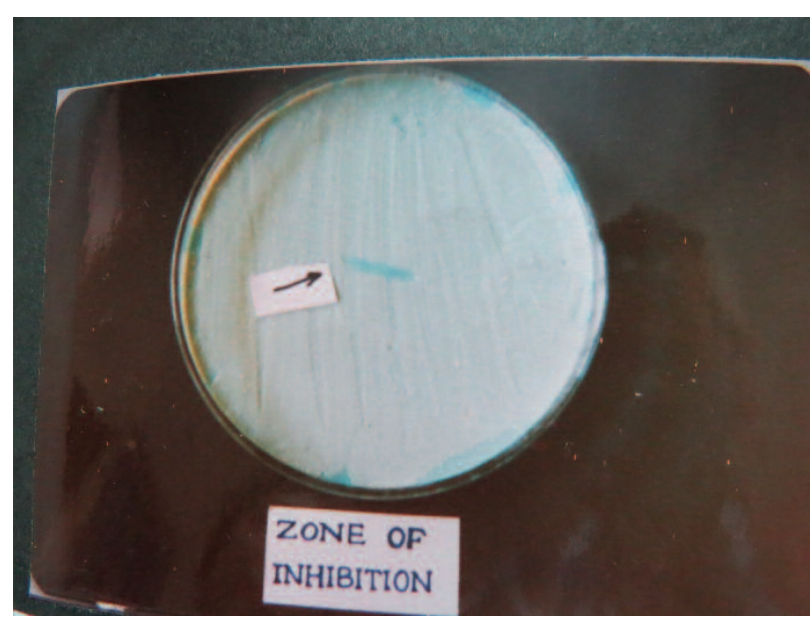

Fig. 2: Zone of inhibition at the site of in-vitro N2 Laser exposure.

Table 2: Incidence of resistance to anti-TB drugs

\begin{tabular}{lc}
\hline \multicolumn{1}{c}{ Drug } & Resistance (\%) \\
\hline INH & 55.7 \\
\hline Streptomycin & 41.8 \\
\hline Pyrazinamide & 40 \\
\hline Ethambutol & 20 \\
\hline Rifampicin & 21.5 \\
\hline Sodium PAS & 13 \\
\hline Thiocetazone & 6 \\
\hline Kanamycin & 9 \\
\hline
\end{tabular}

The N2 laser was fabricated by Centre for Advanced Technology, Indore, M.P., India and provided to Choithram Hospital and Research Centre for therapeutic trials. The fiber was disinfected with Cidex after use for the patient.

\section{Results}

Five hundred sixty seven isolates of $M$. tuberculosis were subjected to drug susceptibility testing by proportion method. Incidence of resistance to various anti-TB drugs is shown in Table 1. Rifampicin resistance was 21.5\% and reflects the MDR strains. Simultaneous resistance to various drugs is depicted in Table 2 . Only $12.8 \%$ of the isolates were sensitive to all the drugs.

In-vitro inhibition of $M$. tuberculosis was noted when it was exposed to the N2 laser for $10 \mathrm{~min}$. The experiment confirmed the inactivating effect of N2 laser for M. tuberculosis (Fig. 2)

For in-vivo study 96 pulmonary tuberculosis cases were selected which were suspected MDR suspected cases since they did not respond well to Anti tubercular treatment.

Of 96 patients, 75 (78\%) were males and 21 (22\%) were females. The age distribution of patients is shown in Table 3. Forty three (70 \%) were between 21-30 years. The following results were noted after nitrogen laser treatment; Clinical improvement occurred in $90 \%$ of the patients with in two days of N2 laser therapy, toxicity and severity was reduced appetite improved and there was marked sense of wellbeing. Sputum conversion to AFB smear negative was achieved in 75\% within a few days (4-15 days). Radiological improvement appeared in $60 \%$, of the

Table 3: Case distribution

\begin{tabular}{cc}
\hline Age & Number \\
\hline $15-20$ & 7 \\
\hline $21-30$ & $43(\mathrm{Max} 70 \%$ \\
\hline $31-40$ & 25 \\
\hline $41-50$ & 12 \\
\hline $51-60$ & 6 \\
\hline $61-70$ & 3 \\
\hline
\end{tabular}


patients. Cavities often disappeared within 8-12 weeks. Some of the cases are discussed below.

Case 1. A 24-year-old male who presented with cough, hemoptysis, and loss of weight. He was under treatment with 4 drugs for 4.5 months. Sputum AFB positivity suggested that he was resistant to anti-TB drugs. A skiagram of his chest revealed a large cavity in the right upper zone. Intracavitar N2 laser was given for 10 minutes. After 10 week time the cavity had completely disappeared. The patient was asymptomatic; sputum AFB negative indicating complete clinical and radiological cure. Follow up to date showed no recurrence (Fig. 3 before N2 Laser exposure; Fig. 4 after N2 laser exposure).

Case 2. A 19-year old male with cyanotic congenital heart disease with tricuspid atresia. He had cough, fever, and hemoptysis for 1 year; received anti-TB drugs but sputum AFB positive $(++)$ for 2.5 months. Xray of the chest showed the cavity in right mid zone. Intra-cavitary N2 laser was given for 10 minutes. The patient became asymptomatic and sputum AFB negative. The cavity disappeared in 6 weeks. There has been no recurrence during 3 years follow-up.

Case 3. Twenty one year-old female. She had 3 years of irregular treatment and was very sick with cough, fever, loss of weight and hemoptysis, sputum was AFB positive $(++++)$.Her lung cavity was irradiated with N2 laser for $10 \mathrm{~min}$. She had very good symptomatic relief the very next day; 48 hours later sputum showed 3-4
AFB per HPF and became AFB negative in 14 days. She had marked clinical and radiological improvement and was sputum AFB negative. She stopped treatment and became sputum AFB positive (after 2 months) Nitrogen laser irradiation was repeated and she became AFB negative; the cavity fibrosed and closed in 6 weeks. In 4 years follow-up there has been no recurrence

Case 4. A 46-year old female. She had had irregular treatment for 20 years. She had tried all possible drugs in various combinations. She had cough hemoptysis and sputum AFB was positive $(++++)$. An x-ray of the chest revealed multiple large cavities on both sides of the lungs combined with heavy infiltration. She had two sittings of intracavity N2 laser for 10 min each in lower right side. She had good clinical improvement: no fever, no hemoptysis, only a mild cough, and the sputum AFB negative. $\mathrm{X}$ ray confirmed that cavity in right lower side disappeared. However, she did not return for follow-up.

Case 5. A 32-year-old female. She had had cough expectoration for 1 year and had been on the regular four drugs (rifampicin, INH, streptomycin, and ethambutol) for 6 months. Her sputum was AFB positive and a skiagram of the chest revealed a large cavity in the right upper zone. Intra-cavitary N2 laser was given for 10 min. The cavity disappeared in 5 weeks.

Regular follow-up revealed complete clinical and radiological cure. Her sputum remained AFB negative and treatment was stopped after 9 months.

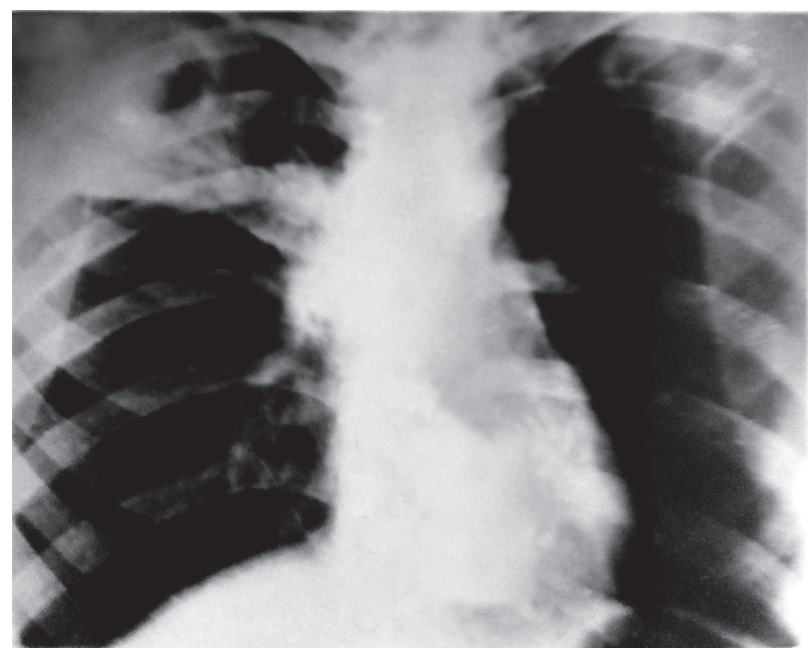

Fig. 3: Chest X-ray film of Case No. 1 before N2 Laser.

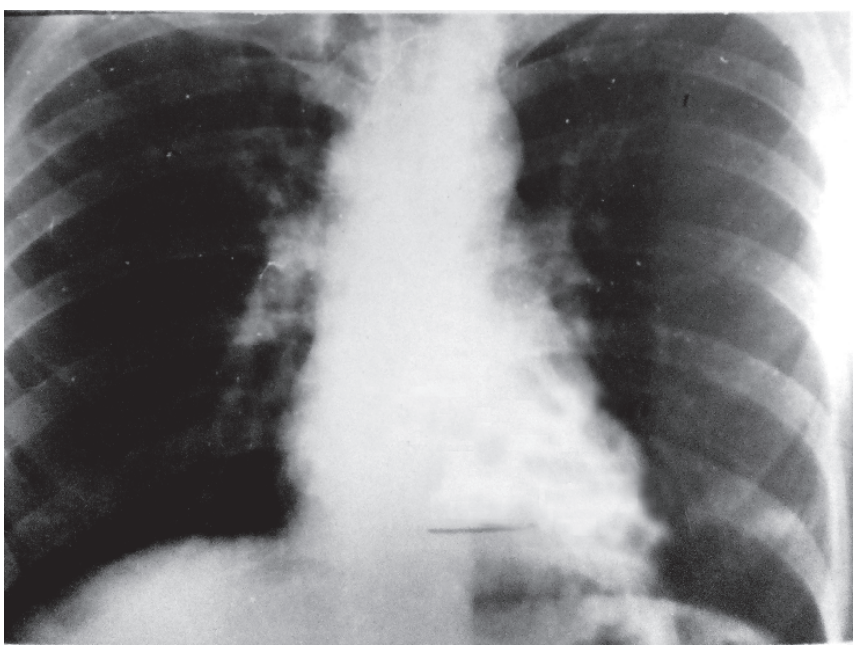

Fig. 4: Chest X-ray film of Case No. 1 after 10 weeks of N2 Laser exposure. 


\section{Discussion}

Among infectious diseases, tuberculosis has perhaps the highest morbidity and mortality. Its partnership with HIV has threatened the developed World as well and the growing multiple drug resistance has added to its menace ${ }^{1}$ ). The first author (Dr. N.S, Bhagwanani) had witnessed the use of N2 laser therapy for TB patients in early nintees in Tashkent. ${ }^{15)}$ However, scientific rationale for the use of $\mathrm{N} 2$ laser was rather poor at the time. In the present communication, direct killing of $M$. tuberculosis on exposure to N2 laser was evident. Macrophage system forms the key system in the pathology and immunity to tuberculosis and a quiescent macrophage is incapable of killing phagocytosed macrophages. ${ }^{16)}$ The activation of macrophages may be necessary to enhance the killing of intracellular organisms. ${ }^{5)}$ The accelerated killing of mycobacteria by the monocytes exposed to N2 and He-Ne laser was documented by the work at our end ${ }^{4)}$. The above background supports the use of N2 laser as an adjunct for the treatment of tuberculosis.

Clinical improvement was seen for $90 \%$ of the patients in the present study. Sputum conversion to negative smear was achieved in 75\% cases within 4-15 days. Radiologically cavities disappeared in 8-12 weeks. Thus, the N2 laser therapy was seen to be a remarkable adjunct to chemotherapy in the present study. Thick fibrosed wall of the cavities may hinder the transfer of anti-TB drugs within the lung cavities and N2 through quartz fiber could have a therapeutic advantage. The complete mechanism of action of N2 laser in the therapy needs to be uncovered but the present data strongly supports its utility as an adjunct to chemotherapy.

The present study is a pilot study and muticentric trials are required before the regular acceptance of the modality.

\section{Conclusions}

MDR prevalence among TB patients on chemotherapy was $21.5 \%$ at our end. The N2 laser was shown to have direct microbicidal effect and it was tried as intra-cavitary irradiation for 96 pulmonary tuberculosis cases not responding to chemotherapy. Clinical improvement seen in $90 \%$ subjects, sputum AFB negativity occurred in $75 \%$ cases within $4-15$ days. The cavities often disappeared in 8-12 weeks. The N2 laser therapy had a remarkable success as an adjunct to chemotherapy.

7: Bermudez LE, Young LS (1988): Tumor necrosis factor, alone or in combination with $1 \mathrm{~L}-2$, but not IFN-gamma, is associated with macrophage killing of Mycobacterium avium complex. J Immunol., 140: 3006-3013.

8: Rose RM, Fuglestad JM, and Remington L (1991): Growth inhibition of Mycobacterium avium complex in human alveolar macrophages by the combination of recombinant macrophage colony-stimulating factor and interferon-gamma. Am J Resp Cell Mol., 4: 248-254.

9: Denis, M (1991): Tumor necrosis factor and granulocyte macrophage-colony stimulating factor stimulate human macrophages to restrict growth of virulent Mycobacterium avium and to kill avirulent $M$. avium: killing effectors mechanism depends on the generation of reactive nitrogen intermediates. J Leukocyte Biol., 49: 380-387.

10: Bermudez, L.E., Young, L.S. (1990): Recombinant granulocyte-macrophage colony stimulating factor activates human macrophages to inhibit growth or kill Mycobacterium avium complex J Leukocyte Biol., 48: 67-73. 
11: Denis M, Gregg EO (1990): Recombinant tumour necrosis factor - alpha decreases whereas recombinant interleukin-6 increases growth of a virulent strain of Mycobacterium avium in human macrophages. Immunology, 71: 139-141.

12: Koneman EW, Allen SD, Janda WM, Schreckenberger PC, Washington Jr CM (1997): Color atlas and textbook of diagnostic microbiology, 5th ed., Philadelphia: JB Lippincott.

13: Kubica GP, Dye WE, Cohn ML, Middlebrook G (1963): Sputum digestion and decontamination with N-acetyl-L-cysteine sodium hydroxide for culture of mycobacteria. Am Rev Respir Dis., 87: 775-
779.

14: Canetti G, Froman F, Grosset J, Hauduroy P, Langerova M, Mahler HT et al., (1963): Mycobacteria: laboratory methods for testing drug sensitivity and resistance. Bull World Health Organ, 29: 565-578.

15: Eshankhanov M, Khodzhaeva MI, Takhirov ON, Priluk KP (1989) : Nitrogen laser in the therapy of lung destructive tuberculosis. In : Proceedings of the International Conference, Tashkent, part 3, p 206

16: Wallis RS, Ellner JJ (1994): Cytokines and Tuberculosis. J Leukocyte Biol., 55: 676-681.

\section{[Acknowledgement]}

The authors are thankful to Dr. D.P Bhawalkar, Ex-Director, and Centre for Advanced Technology, Indore and his team for proving the Nitrogen laser. Thanks are due to National Laser Programme for funding the work and to the Management of Choithram Hospital and Research Centre for the infra structural support. 